gänglicher Forschung andererseits zu unterscheiden sei; dieser öffentliche Zugang sei in Deutschland im Vergleich zu den USA noch vergleichsweise gut. Solche praktische, organisationsbezogene Beratung hatte vor allem der Präsident der LfK, Dr. Thomas Hirschle, eingefordert, speziell mit Blick auf aktuelle Entwicklungen bei den Mediendiensten. Daß es zu unmittelbarer Rückkopplung zwischen Medienforschung und Senderproduktion kommen kann, zeigte das Beispiele des Kinderkanals, worüber Karin Richter, Professorin an der Pädagogischen Hochschule Erfurt, berichtete.

Wie erfrischend und treffend eine polemische Zeitdiagnose sein kann, demonstrierte auf dem Podium Dr. Ingo Hermann, lange Zeit im ZDF auch mit Kindersendungen befaßt. Vom "Verschwinden der Kindheit" (so vor längerer Zeit Neil Postman) könne gar keine Rede sein, es sei nachgerade eine Infantilisierung der Erwachsenenwelt festzustellen, was Hermann u.a. an Entzugserscheinungen festmachte, wenn Handybesitzer nicht ständig die Trivialkoordinaten ihrer Bewegung durch Raum und Zeit durchgeben könnten. Diese Kategorien verändern sich, was so recht erst in der historischen Dimension deutlich werde, worauf Hartmut Eggert, Professor für Germanistik an der FU Berlin, hinwies. Freilich bleiben solche zeitbezogenen wie übergreifenden Diagnosen auf die Forschung zurückverwiesen, womit auch der letzte Teilnehmer auf dem Podium nicht unerwähnt bleiben soll, der schon eingangs genannt wurde, Walter Klingler, der zusammen mit Gunnar Roters (der den zweiten Tag moderierte) und Maria Gerhards die Tagungsorganisation innehatte, die man insgesamt doch als recht gelungen einstufen darf.

Trotzdem lasse ich es mir nicht nehmen, anhand dieses Podiumsgespräches abschließend noch einmal auf mediale Randbedingungen hinzuweisen: Die Abfolge 'Moderatorfrage Mikro ergreifen - Statement abliefern' führt doch Bedingungen der Kanalisierung und Segmentierung in ein Kommunikationsgeschehen ein, das ohne Technik ganz anders und viel lebendiger verlaufen würde, dessen bin ich mir sicher. Könnte nicht auch dies ein Aspekt von Infantilisierung sein, daß wir meinen, alles speichern zu müssen? Und sollten wir uns nicht wenigstens gelegentlich von solchem Zwang frei machen, auch auf die Gefahr hin, daß dann nur die Dabeigewesenen davon erzählen (oder darüber schreiben) können?

$\ll$

\section{Workshop "Von der Informa- tions- zur Wissensgesellschaft. Demokratie - Partizipation - Technikfolgenbeurteilung"}

\section{Tagungsbericht von K. Friedrich, Branden- burgische Technische Universität Cottbus, und P. Machleidt, Akademie der Wissen- schaften der Tschechischen Republik}

Die Europäischen Akademie zur Erforschung von Folgen wissenschaftlich-technischer Entwicklungen Bad Neuenahr-Ahrweiler $\mathrm{GmbH}$ und das Zentrum für Wissenschafts-, Technikund Gesellschaftsstudien beim Institut für Philosophie der Akademie der Wissenschaften der Tschechischen Republik hatten zum Workshop "Von der Informations- zur Wissensgesellschaft. Demokratie - Partizipation - Technikfolgenbeurteilung" vom 3. bis 5. Februar 1999 nach Prag eingeladen. Dieser Einladung waren mehr als 35 Interessenten verschiedener Institutionen aus neun Ländern Europas gefolgt. ${ }^{1}$ Dieser Workshop war der zweite im Rahmen des Projekts "Technikfolgenbeurteilung und Wissenschaftsethik in Ländern Mittel- und Osteuropas" der Europäischen Akademie GmbH. Er diente einerseits als Abschluß des auf zwei Jahre befristeten Projekts ${ }^{2}$ und andererseits der Weiterentwicklung der zielgerichteten Kooperationsbeziehungen der Europäischen Akademie mit Wissenschaftseinrichtungen vor allem in Polen, Tschechien und Ungarn. In diesem Sinne war der Workshop auch Ausgangspunkt für die Diskussion zukünftiger Formen und Inhalte der Zusammenarbeit.

Das Ziel des Workshops war ein mehrfaches. Als erstes sollten durch die verschiedenen Länder und Institutionen Sichten und Problemlagen zu dem gewählten Thema dargelegt und so für eine vergleichende Betrachtung aufbereitet werden. Zweitens sollten Rückkopplungseffekte hinsichtlich der gegenseitigen Informations- und Erfahrungsweitergabe erzielt werden. Darauf aufbauend sollte drittens über zukünfti- 
ge mögliche gemeinsame Vorhaben und Projekte beraten werden.

Damit richtete sich das Anliegen des Workshops auf die themen- und entscheidungsbezogene Bündelung des verfügbaren Wissens, vor allem technischer, sozialer, politischer und ethischer Art, und auf das Erkennen von Technisierungsfolgen für das individuelle und soziale Leben, besonders bezogen auf die Voraussetzungen wie die Konsequenzen der umfassenden Nutzung der neuen informationstechnischen Möglichkeiten sowie deren Beurteilung hinsichtlich ihrer Akzeptabilität und Wünschbarkeit. So ging es insbesondere sowohl um Ergebnisse und (Forschungs-) Hypothesen als auch um das Kennzeichnen offener Fragen und ungelöster Probleme. Auf der anderen Seite handelt es sich vor allem um die Begründung von handlungsrelevanten Zwecken und Zielen, um die Charakterisierung technikbezogener Normen- und Wertkonflikte einschließlich deren Grundlagen und möglicher Lösungsstrategien sowie das Verdeutlichen von Handlungsoptionen mit ihren jeweiligen Chancen und Gefahren.

Mit dem gewählten Thema "Von der Informations- zur Wissensgesellschaft" wurde eine Problematik in den Mittelpunkt gerückt, die infolge der damit verbundenen vielfältigen technischen, sozialen, politischen, ökonomischen und ethischen Facetten, Implikationen und Herausforderungen eine Gestaltungsaufgabe ersten Ranges für alle Industrieländer darstellt, betonte zur Eröffnung Herr Professor Zahradnik, Präsident der Akademie der Wissenschaften der Tschechischen Republik. Er begrüßte gleichzeitig die Durchführung des internationalen Workshops in Hause der Akademie der Wissenschaften in Prag als wichtiges Zeichen der Forschungskooperation zwischen beiden Akademien und den anderen Institutionen, handele es sich doch um die erste wissenschaftliche Veranstaltung dieser Art seit Beginn des Transformationsprozesses. In seinem Öffentlichen Vortrag im Rahmen der Eröffnungsveranstaltung des Workshops ging Herr Professor Salomon (CNAM, Paris, Frankreich) vor mehr als fünfzig Zuhörern auf den Zusammenhang von Wissenschaft, Demokratie und technischen Risiken ein und hob besonders die Bedeutung der Partizipation bei der Bewältigung technologischer Risiken hervor.
Die Erörterung der Schwerpunkte des Programms "Das Konzept", "Die Einsatzfelder" und "Die Beurteilung" eröffneten der Direktor der Europäischen Akademie Bad NeuenahrAhrweiler GmbH, Herr Professor Gethmann, und der Leiter des Zentrums für Wissenschafts-, Technik- und Gesellschaftsstudien beim Institut für Philosophie der Akademie der Wissenschaften der Tschechischen Republik, Herr Professor Tondl. Sie hoben nochmals den Stellenwert der Beratung als wichtiges Vorhaben zur Generierung wie Weiterführung gemeinsamer fruchtbringender Projekte von Wissenschaftlern aus verschiedenen Teilen Europas auch als Beitrag zu einem gemeinsamen Europa - hervor.

Im Schwerpunkt I "Das Konzept" (Leitung: Professor Banse) wurden zunächst das Konzept und der Begriff der Informationsgesellschaft von Herrn Bechmann (ITAS) zur Diskussion gestellt und sodann anhand des Transformationsprozesses der Gesellschaft in Tschechien als Gegenstand konkreter Betrachtungen von den Herren Dr. Provazník, Dr. Filácek und Dr. Machleidt (Tschechische Akademie der Wissenschaften) eingeführt. Gotthard Bechmann ging davon aus, daß die Möglichkeit der Feststellung der Veränderung von Strukturen die Thematisierung des sozialen Wandels der Gesellschaft als Informationsgesellschaft möglich macht, deren Beschreibung nicht bei technischen Prozessen stehen bleiben darf. "Informativer sind Theorien, die von der zentralen Annahme ausgehen, daß die Produktion, Verteilung und Verarbeitung von Informationen zu einer konstitutiven Voraussetzung der sozialen Systeme in den entwickelten Industriestaaten geworden ist", hatte er in seinem Abstrakt formuliert. Vorgestellt wurden folgende Konzepte, die den von der Informationstechnik eingeleiteten sozialen Wandel erfassen: Informationsgesellschaft als Informationswirtschaft; als postindustrielle Gesellschaft, als Ende der industriellen Arbeits- und Massenproduktionsgesellschaft sowie als Wissenschaftsgesellschaft. Alle basieren auf einem undefinierten Informationsbegriff. Damit begründete er sein Konzept, die Informationsgesellschaft vom Begriff der Information im Anschluß an Luhmann her zu erklären und zu begründen. Die anschließende Diskussion zeigte die Notwendigkeit einer Festlegung einer gemeinsamen Herangehens- 
weise an die Bestimmmung des Wesens und der Probleme der Informationsgesellschaft zur Verifizierung der gesellschaftlichen, kulturellen und technischen Dimension. Der Beitrag der Kollegen der Tschechischen Akademie charakterisierte den Prozess der Transformation der Gesellschaft in Tschechien aus dem Blickwinkel der Anforderungen weniger der Informations- als mehr der Wissensgesellschaft. Sie betonten die wachsende Bedeutung technologischer Innovationen und die Notwendigkeit der Ausnutzung vorhandener Ressourcen besonders auf dem Gebiet der Bildung und der Wissenschaft für die Entwicklung in ihrem Land. Das "Modell der Wissenschaftsgesellschaft" als Bestandteil gesellschaftlicher Entwicklungsmodelle weiter zu konkretisieren sei ein wichtiges Moment interdiszplinärer Forschungen in einer Situation, die auch in Tschechien durch den Abbau von Forschungs- und Wissenschaftsförderung gekennzeichnet ist. Die tiefgreifende Transformation des sozialen, politischen und ökonomischen Systems war und ist bis heute nicht problemlos. Damit ist mehr als nur die einschneidende Reduktion der Forschungsbasis und insbesondere der angewandten Forschung in der Tschechischen Republik gemeint. Es sind, wie gesagt, auch soziale, ökonomische und ethische Faktoren mit betroffen. Zwar wurden institutionelle Strukturen verändert, aber in vielen Aspekten haben sich längst überholte Bewertungskriterien bei der Beurteilung der Industriegesellschaft wieder etabliert. Die Forschungs- und Entwicklungsaktivität sollte daher zukünftig an einer weiteren Entfaltung bzw. an der Schaffung solcher Initiativen zu mehr Innovationen stärker als bisher teilhaben.

Im Schwerpunkt II "Die Einsatzfelder" (Leitung: Professor Gorokhov, Russische Akademie der Wissenschaften) wurden unterschiedliche Sichten auf Folgen der Informationstechnik vorgestellt. ${ }^{3}$ Herr Professor Hronszky (TU Budapest) eröffnete die Aussprache mit der Sicht auf den Wandel der Technikfolgenbeurteilung, in dem unter anderem die Rolle und Bedeutung der Experten eine Veränderung erfährt. Dies umfasse erstens zum einen die wachsenden Anforderungen an Expertisen infolge ihrer (oft wirtschaftlichen) Bedeutung, sowie zum anderen deren Möglichkeit (bzw. Unmöglichkeit), sowohl Komplexität zu erfassen als auch Risiken und Unsicherheiten techni- scher Entwicklungen vorauszusagen. Als zweites ergibt sich daraus die Frage, wer als Experte $\mathrm{zu}$ welchen Fragen herangezogen wird. Drittens erfordert die Notwendigkeit der Partizipation der Öffentlichkeit an Entscheidungen über technische Entwicklungen die Herausbildung eines Prozesses des "sozialen Lernens", eine Aufgabe, die auch für Ungarn notwendig ist. Die sich anschließende Diskussion wies auf die Notwendigkeit der Partizipation und der Bedeutung der Expertisen im Umfeld der Betrachtung der Folgen wissenschaftlich-technischer Entwicklungen hin und hob gleichzeitig das Zusammentragen der Erfahrungen in unterschiedlichen Ländern und aus unterschiedlichen Projekten hervor. Gegenstand der Ausführungen von Herrn Professor Kiepas (Schlesische Universität Katowice) war der "Forschungsund Entwicklungsstand der Informationsgesellschaft in Polen". Er wandte sich besonders den Nutzungen und Anwendungen des Internet in Polen zu, welches heute noch von "einer kleinen Elite genutzt wird", aber darauf hinweist, daß demnächst wesentliche Veränderungen zu erwarten sind. Die 1995 gegründete Polnische Gemeinschaft der Internetbenutzer und das Zentrum für Informationsgesellschaft an der Schlesischen Universität in Katowice unter Leitung von Professor Zacher sind besonders um theoretische Reflexionen auf diesem Gebiet bemüht. Als Grundproblem erweist sich zunehmend, daß es keine Konzepte für die Entwicklung und Einführung der modernen Informations- und Kommunikationstechnologien in Polen gibt. Professor Kiepas beschrieb neben optimistischen, pessimistischen und neutralen Haltungen zum Internet dessen ethische Dimension und seine Bedeutung für und in der Ausbildung in Polen. Mit diesem Beitrag wurde eine Facette zukünftiger gemeinsamer Arbeit sichtbar, die Bedeutung der Informations- und Kommunikationstechnologien für den Bildungsbereich und die Notwendigkeit der kritischen Betrachtung der zunehmenden Nutzung des Internet in den verschiedenen Ländern.

Daran schloß sich der Beitrag von Herrn Dr. Ulrich (Bundesamt für Sicherheit in der Informationstechnik, Bonn) an, der die Bedeutung der informationstechnischen (IT-) Sicherheit nicht nur in ihrer technischen, sondern vor allem in ihrer sozialen und kulturellen Dimension charakterisierte. IT-Sicherheit betrifft nicht 
nur die Minderheit der derzeitigen Nutzer moderner Kommunikationsmedien, sondern auch jene, die von diesen Entwicklungen zunehmend betroffen sind. Damit wird die Notwendigkeit der Entwicklung einer Sicherheitskultur und Medienkompetenz deutlich. Mit dem Gesetz über digitale Signaturen, das - so wurde in ihm festgelegt - nach wenigen Jahren einer Überprüfung unterzogen werden wird, ist vielleicht ein Beispiel des neuen Umgangs mit Sicherheit gegeben.

Eine systemtheoretische Sicht, vor allem die Bedeutung der Nutzung der Methoden und Techniken aus dieser Perspektive, wurde in dem Beitrag von Herrn Professor Vlcek (Technische Univeristät Prag) vorgestellt.

Der Übergang zum dritten Schwerpunkt "Die Beurteilung" (Leitung: Frau Professor Eijndhoven, Rathenau-Institut, Niederlande) wurde durch Herrn Professor Tamás (Ungarische Akademie der Wissenschaften) mit der Betrachtung der Entwicklung der Informationsgesellschaft unter den nationalen Bedingungen in Ungarn aus unterschiedlicher Sicht hergestellt. Die gesellschaftlichen Wandlungen zur Informationsgesellschaft scheinen in den "postsozialistischen Länden" ohne nationale Strategie und ohne Berücksichtigung der jeweiligen kulturellen Bedingungen der Länder zu verlaufen. In Ungarn zeichnet sich ein Wechsel von politischen $\mathrm{zu}$ ökonomischen Prämissen ab. Damit wird zugleich das Forschungsfeld eröffnet, in dem kulturelle Kontexte der Informationstechnik, deren Anwendungsbedingungen und deren erreichte Wirkungen interessieren. Herr Dr. Grunwald (Europäische Akademie) stellte grundsätzliche aktuelle Aspekte der Diskussion in Wissenschaft, Politik und Öffentlichkeit über Dimensionen der Wissensgesellschaft vor. Diese wurden am Konzept der Technikfolgenbeurteilung und der Technikgestaltung diskutiert. Aspekte der Rationalität und Partizipation sowie deren Bedeutung für gesellschaftliche Lernprozesse wurden besonders betont. Herr Professor Tondl schließlich spannte mit "Wissen und Wertvoraussetzungen der Technikfolgenbeurteilung" den Bogen von den Problemen und Anforderungen der Wissensgesellschaft zur besonderen Bedeutung von internationalen interdisziplinären Projekten, welche sich besonders auf die humane, kulturelle und soziale Dimension richten. Es zeigt sich auch, daß es - wie Professor Tondl betonte - bisher nicht gelungen ist, voll und ganz die menschlichen Ressourcen auszuschöpfen, insbesondere die intellektuellen Fähigkeiten, die in den mitteleuropäischen Zentren noch immer tief verwurzelt sind und eine große Tradition haben. Sie werden oft durch andere Vorstellungen und Werte, die an rein kommerziellen, medial präformierten oder seit jeher propagierten Informationsmustern orientiert sind, überformt.

Die Abschlußdebatte (Leitung: Herr Professor Banse) bestätigte das Konzept der Veranstalter, daß das behandelte Problemfeld notwendig Momente der internationalen Zusammenarbeit erfordert und ermöglicht. Zum einen sind das das Konzept der (einer?) Informationsund der Wissensgesellschaft in nationaler und internationaler Dimension sowie neben ihren technischen vor allem deren kulturelle, ethische, soziale und ökonomische Fragestellungen. Dazu gehören weiterhin Fragen der Partizipation und der Demokratie, die etwa im Hinblick auf Internet und IT-Sicherheit weiter entwickelt werden müssen. Schließlich ist ein wichtiger Punkt, daß es zunehmend der Aufklärung der von der Informationstechnik "Betroffenen" bedarf. Daraus können sich alsbald neue Forschungskooperationen und vielleicht auch ein nächster Workshop ergeben. Konkretisierende Vorstellungen gab es bereits am "Rande" dieser wissenschaftlichen Veranstaltung.

Als Teilnehmer des Workshops, der vor allem von der konzeptionellen Arbeit und dem Engagement von Herrn Dr. Machleidt auf tschechischer Seite und von Herrn Professor Banse und Herrn Dr. Langenbach seitens der Europäischen Akademie getragen wurde, bleibt nicht nur die Erwartung auf weitere gemeinsame Vorhaben des charakterisierten Problemfeldes, sondern vor allem die baldige Veröffentlichung von Vorträgen und Diskussionsbeiträgen in der "Schriftenreihe" der Europäischen Akademie.

\section{Anmerkungen}

1 U.a. von der Karlsuniversität Prag, Technischen Universität Prag, dem PIAS, der Universität Pardubice und der Westböhmischen Universität Pilsen, der Grant Agentur und dem Ministerium für Verteidigung (Tschechische Republik), der Schlesischen Universität Katowice und der Hochschule für Sozialwissenschaften 
Tychy (Polen), der Ungarischen Akademie der Wissenschaften und der Technischen Universität Budapest (Ungarn), der Akademie der Wissenschaften in Rußland, dem ITAS, dem BSI, der BTU Cottbus, der Fachhochschule Merseburg, dem Transferzentrum für angepaßte Technologien $\mathrm{GmbH}$ Rheine und der Friedrich-Ebert-Stiftung, Bonn, aus Deutschland, sowie vom Centre Science, Technologie et Société, Paris, Frankreich, und dem Rathenau-Institut in den Niederlanden.

2 Der 1. Workshop fand am 22. und 23. Januar 1998 in Bad Neuenahr-Ahrweiler statt. Vgl. dazu Banse, G.: Workshop "Technikfolgenbeurteilung und Wissenschaftsethik in Ländern Mittel- und Osteuropas". In: TA-DatenbankNachrichten, Nr. 2/1998, S. 97-101); zu ersten Ergebnissen, die sich auf den Workshop und das Projekt insgesamt beziehen, vgl. Banse, G. (Hrsg.): Technikfolgenbeurteilung und Wissenschaftsethik in Ländern Ostmitteleuropas. 2 Teile. Bad Neuenahr-Ahrweiler 1998 ("Graue Reihe", Bd. 10); siehe auch Banse, G.: Technikfolgenbeurteilung in Ländern Mittel- und Osteuropas - erste Ergebnisse eines Projekts. In: TA-Datenbank-Nachrichten, Nr. 4/1998.

3 Für diesen Schwerpunkt waren zwei weitere Vorträge im Programm vorgesehen, die Referenten mußten jedoch kurzfristig absagen: Herr Professor Schienstock (Work Research Centre, Universität Tampere, Finnland), "Informationstechnologien und organisationeller Wandel. Das Konzept der technologischen Praxis" sowie Herr Professor Kornwachs (BTU Cottbus) "Daten - Information - Wissen: Versuch einer technologischen Aufklärung".

\section{Kontakt}

Prof. Dr. Gerhard Banse

Universität Potsdam Institut für Philosophie /

Europäische Akademie Bad Neuenahr-Ahrweiler

$\mathrm{GmbH}$

Tel.: +49-(0)331-977 2242 und

Tel.: +49-(0)2641-754300

Fax: +49-(0)331-977 2092 und

Fax: +49-(0)2641-7543 20

E-mail: banse@rz.uni-potsdam.de und

E-mail: europaeische. akademie@dlr.de
Dr. Christian Langenbach

Europäische Akademie Bad Neuenahr-Ahrweiler $\mathrm{GmbH}$

Tel.: +49-(0)2641-7543 11

Fax: +49-(0)2641-7543 20

E-mail: christian.langenbach@dlr.de

Dr. Petr Machleidt

Zentrum für Wissenschafts-, Technik- und Gesellschaftsstudien beim Institut für Philosophie der Akademie der Wissenschaften der Tschechischen Republik, Prag

Tel.: +420-(0)2-24212108

Fax: +420-(0)2-2313882

E-mail: stsscz@cesnet.cz 